\title{
High harmonics of the cyclotron resonance in microwave transmission of a high-mobility two-dimensional electron system
}

\author{
M. L. Savchenko ${ }^{10},{ }^{1,2, *}$ A. Shuvaev ${ }^{\circ},{ }^{3}$ I. A. Dmitriev, ${ }^{4,5}$ A. A. Bykov ${ }^{(1,},{ }^{1,2}$ A. K. Bakarov ${ }^{1,2},{ }^{1,2}$ \\ Z. D. Kvon, ${ }^{1,2}$ and A. Pimenov ${ }^{3}$ \\ ${ }^{1}$ Rzhanov Institute of Semiconductor Physics, 630090 Novosibirsk, Russia \\ ${ }^{2}$ Novosibirsk State University, Physics Department, 630090 Novosibirsk, Russia \\ ${ }^{3}$ Institute of Solid State Physics, Vienna University of Technology, 1040 Vienna, Austria \\ ${ }^{4}$ Terahertz Center, University of Regensburg, 93040 Regensburg, Germany \\ ${ }^{5}$ Ioffe Institute, 194021 St. Petersburg, Russia
}

(Received 26 August 2020; accepted 12 January 2021; published 5 February 2021)

\begin{abstract}
We report an observation of magneto-oscillations in transmittance of the circularly polarized microwave radiation through the high-mobility two-dimensional electron system hosted by a GaAs quantum well. The oscillations reflect an enhanced absorption of radiation at high harmonics of the cyclotron resonance and follow simultaneously measured microwave-induced resistance oscillations (MIRO) in the DC transport. While the relative amplitude (up to $1 \%$ ) of the transmittance oscillations appears to be small, they represent a significant (greater than 50\%) modulation of the absorption coefficient. The analysis of obtained results demonstrates that the low- $B$ decay, magnitude, and polarization dependence of the transmittance oscillations accurately follow the theory describing photon-assisted scattering between distant disorder-broadened Landau levels. The extracted sample parameters describe reasonably well the concurrently measured MIRO. Our results provide insight into the MIRO polarization immunity problem and demonstrate that high-precision transmission measurements can be a sensitive probe of high-frequency dissipative effects in high-mobility systems.
\end{abstract}

DOI: 10.1103/PhysRevResearch.3.L012013

\section{INTRODUCTION}

The discovery of microwave-induced resistance oscillations (MIRO) and zero-resistance states in high-mobility two-dimensional electron systems (2DESs) subject to a moderately strong perpendicular magnetic field [1-3] triggered an outbreak of experimental and theoretical research that has led to the observation of a number of interrelated magnetotransport phenomena in various materials and conditions [4-26]. Most of the observed effects have been coherently explained within a unified framework describing the quantum kinetics of electrons in disorder-broadened Landau levels (LLs) in the presence of strong static and alternating electric fields [4,27-39] (for other theoretical proposals, see Refs. [40-43]). In application to MIRO, this quantum description is tightly linked to an enhanced absorption of microwave radiation whenever the photon energy $\hbar \omega$ is close to an integer multiple of the cyclotron energy $N \hbar \omega_{c}[29,31,32,35]$. In a nutshell, such enhancements reflect the maxima of the thermally averaged product $\langle v(\varepsilon) v(\varepsilon+\hbar \omega)\rangle$ of initial and final densities of states for transitions between the disorder-broadened LLs. It

\footnotetext{
*mlsavchenko@isp.nsc.ru

Published by the American Physical Society under the terms of the Creative Commons Attribution 4.0 International license. Further distribution of this work must maintain attribution to the author(s) and the published article's title, journal citation, and DOI.
}

is worth mentioning that, apart from the broadening of LLs, the role of disorder here is to make possible the otherwise dipole-forbidden photon-assisted transitions between distant LLs for $N \neq 1$.

The corresponding complex structure of the cyclotron resonance (CR) line shape including multiple additional resonant features at $\omega=N \omega_{c}, N=2,3, \ldots$, was observed already in the early far-infrared transmission experiments [44], following the theoretical predictions in pioneering works on quantum magnetotransport in 2DESs [45]. However, the direct observation of the resonances associated with photonassisted transitions between distant LLs turned out to be a challenging task in modern high-mobility 2DESs [10,46-49], despite giant MIRO and zero resistance states that are argued to emerge due to these processes in the DC resistance of the microwave-illuminated samples [29,31,32]. As detailed below, the reason for this apparent contradiction is that the shape of the magnetotransmittance and reflectance in the ultrahigh-mobility structures is dominated by the nondissipative dynamical response of nearly free electrons such that even very pronounced (up to $\sim 100 \%$ ) quantum corrections to the dissipative part of the dynamic conductivity, $\operatorname{Re} \sigma(\omega, B) \propto$ $\langle v(\varepsilon) v(\varepsilon+\hbar \omega)\rangle$, become hardly detectable in the transmittance traces. It is thus not surprising that in high-mobility structures such quantum oscillations so far have only been detected in the differential absorption of the 2DES placed in a cavity [50].

In this Letter we demonstrate the possibility to detect multiple harmonics of the $\mathrm{CR}$ in the transmission signal on 


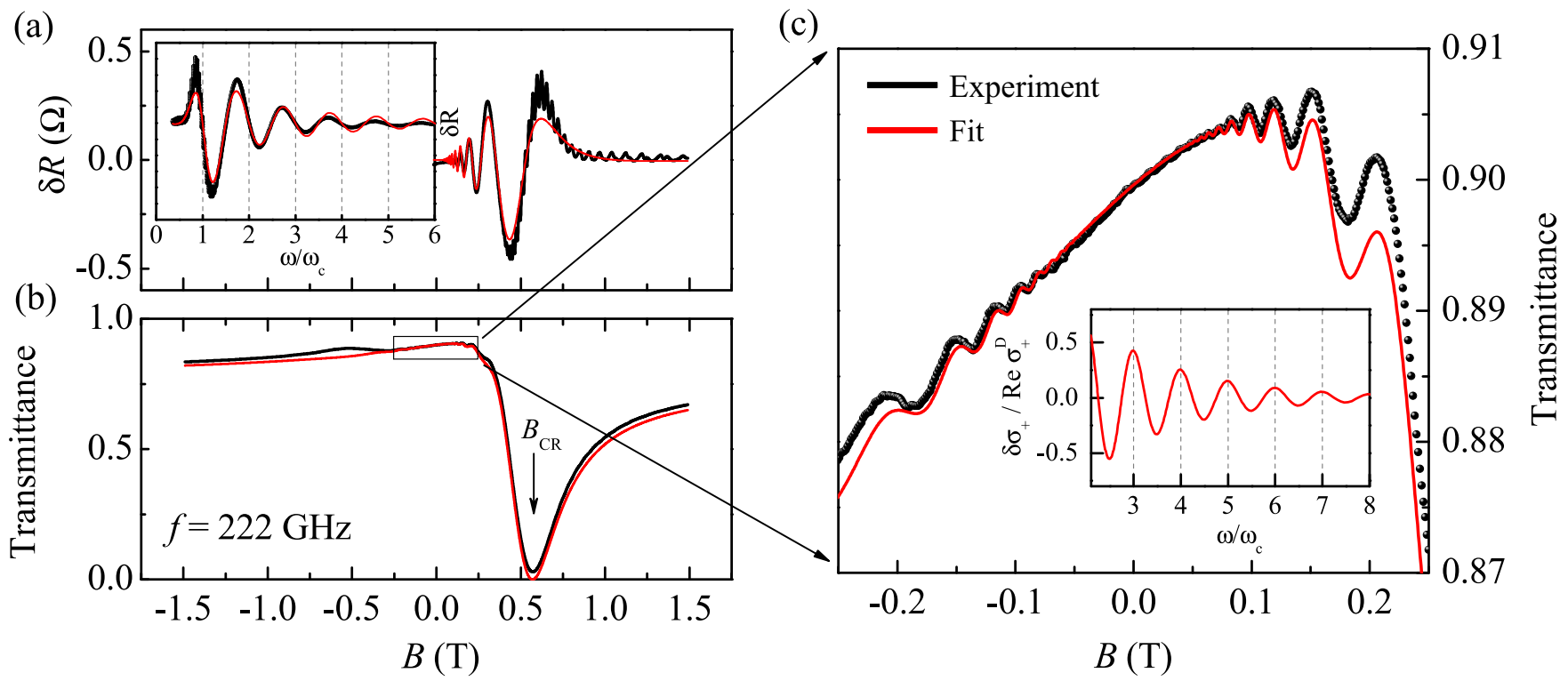

FIG. 1. Magnetic field dependences of (a) photoresistance $\delta R(B)$ and (b) transmittance $\left|t_{+}\right|^{2}(B)$ measured at frequency $f=222 \mathrm{GHz}$ and for the right-hand circular polarization (black). The inset in (a) shows a part of $\delta R(B)$ displaying MIRO plotted against $\omega / \omega_{\mathrm{c}}$. (c) Close-up of $\left|t_{+}\right|^{2}(B)$ in (b), where magneto-oscillations in transmittance are seen at both positive and negative magnetic fields. Red curves show fits calculated according to Eqs. (1)-(3) for the transmittance and Eq. (4) for the photoresistance. The inset in (c) shows the correspondent quantum magneto-oscillations (3) in the dissipative part of the dynamic conductivity $\delta \sigma_{+}$, normalized to the classical Drude background Re $\sigma_{+}^{\mathrm{D}}$, plotted against $\omega / \omega_{\mathrm{c}}$. The period of oscillations in (a) and (c) is the same and yields the effective mass $m=e B / \omega_{\mathrm{c}}=0.068 m_{0}$ of quasiparticles at the Fermi surface, renormalized by electron-electron interactions. On the other hand, the position $B_{\mathrm{CR}}$ of the CR, marked by an arrow in (b), is insensitive to interaction effects by virtue of the Kohn theorem and yields the bare electron mass $m_{\mathrm{CR}}=e B_{\mathrm{CR}} / \omega=0.072 m_{0}$.

a sample that also manifests well-pronounced MIRO in the in situ measured DC resistance. To make this possible, we performed measurements on a sample with moderately high mobility $\mu=2.1 \times 10^{6} \mathrm{~cm}^{2} / \mathrm{V} \mathrm{s}$ and implemented a quasioptical setup with tunable-frequency stable radiation sources which enabled high-accuracy measurements of the absolute transmittance values. To improve the visibility of the quantum magneto-oscillations in transmittance, we further took an opportunity to deliberately modify the shape of magnetotransmittance by fine-tuning the interference effects originating from multiple reflections of the wave in the substrate. With all the above measures, we were able to resolve eight or more periods of magneto-oscillations for both polarities of the magnetic field under the circularly polarized microwave illumination. We show that the low- $B$ decay, the magnitude, and polarization dependence of the observed oscillations accurately follow the theoretical predictions of Ref. [29], being governed by a single fitting parameter which describes the broadening of LLs due to impurity scattering. This parameter also describes reasonably well the low- $B$ decay of the concurrently measured MIRO. Our results demonstrate prospects to use high-precision transmission measurements to probe other high-frequency dissipative effects of high-mobility systems $[40,43,51-54]$ and provide additional insight into the intriguing and controversial issue of polarization immunity of MIRO $[4,10,20,49,55]$.

\section{SAMPLE AND METHODS}

The measurements were carried out on a heterostructure containing a $2 \mathrm{DES}$ in a selectively doped $16-\mathrm{nm}$ GaAs quan- tum well with AlAs/GaAs superlattice barriers grown by molecular beam epitaxy on a GaAs substrate [56-59]. The van der Pauw sample size was $10 \times 10 \mathrm{~mm}^{2}$ and Ohmic contacts at the corners were fabricated by burning $\mathrm{Ge} / \mathrm{Au} / \mathrm{Ni} / \mathrm{Au}$ (see the Supplemental Material for details [60]). After exposure to the room light the electron density and mobility were $n=6.6 \times 10^{11} \mathrm{~cm}^{-2}$ and $\mu=2.1 \times 10^{6} \mathrm{~cm}^{2} / \mathrm{V} \mathrm{s}$, respectively [60]. The sample was irradiated from the substrate side through an 8-mm aperture. Backward-wave oscillators with available frequency $f=\omega / 2 \pi$ in the range between 100 and $1000 \mathrm{GHz}$ were used as stable sources of the normally incident continuous monochromatic radiation. A split-coil superconducting magnet provided the magnetic field $B$ oriented perpendicular to the sample surface. The transmittance through the sample was measured using a He-cooled bolometer. High temporal stability of the whole system including the mounting of the sample allowed us to detect the relative changes of transmittance down to $10^{-4}$ [60,61]. In parallel with the transmittance, the photoresistance $\delta R$ (the difference of the resistance signals in the presence and absence of irradiation) was measured using the double-modulation technique [60]. All presented results were obtained at temperature $T=1.9 \mathrm{~K}$.

\section{RESULTS}

Figure 1 shows a representative example of simultaneously measured photoresistance [Fig. 1(a)] and transmittance [Figs. 1(b) and 1(c)] recorded as a function of perpendicular magnetic field $B$ under $222-\mathrm{GHz}$ circularly polarized radiation. Red lines in Fig. 1 are theoretical fits (explained below) 
to the data shown by black lines and dots. Similar results obtained under $328-$ and $432-\mathrm{GHz}$ radiation are provided in [60].

The photoresistance $\delta R$ in Fig. 1(a) displays pronounced MIRO governed by the ratio $\omega / \omega_{\mathrm{c}}$, where $\omega=2 \pi f$ is the angular radiation frequency and $\omega_{\mathrm{c}}=e B / m$ is the cyclotron frequency. Here $e=|e|$ is the elementary charge and $m$ is the electron effective mass. In the inset, these data are replotted versus $\omega / \omega_{\mathrm{c}}$ (using $m=0.068 m_{0}$, where $m_{0}$ is a free-electron mass) making evident that the observed magneto-oscillations accurately reproduce the established period and phase of MIRO, with nodes at integer $\omega / \omega_{\mathrm{c}}$.

The focus of this work is on the magnetotransmittance. The analysis below shows that it is dominated by strong metallic reflection from the 2DES leading to a strong dip in the region of CR, marked by the arrow in Fig. 1(b). The transmission is also significantly affected by multiple reflections of the electromagnetic wave in the dielectric slab between back and front interfaces of the sample. The associated Fabry-Pérot interference is responsible for an asymmetric line shape of the CR dip in transmittance. For our particular choice of microwave frequency $f=222 \mathrm{GHz}$, the interference produces an almost flat shoulder at $|B| \lesssim 0.2 \mathrm{~T}$. A magnified view of this region, shown in Fig. 1(c), reveals the presence of magneto-oscillations which closely resemble the $\omega / \omega_{\mathrm{c}}$ oscillations seen in $\delta R$. Despite the relatively low amplitude of magneto-oscillations in transmittance and their exponential decay towards low $B$, we are able to clearly detect multiple oscillation periods for both polarities of the magnetic field. Below we demonstrate that these oscillations are in excellent agreement with theoretical predictions of Ref. [29] [see the red line in Fig. 1(c)] and can therefore by attributed to an enhanced absorption at integer $\omega / \omega_{\mathrm{c}}$ due to resonant photonassisted transitions between distant disorder-broadened LLs.

\section{ANALYSIS}

We model the measured transmittance using the expression [44,49]

$$
\left|t_{ \pm}\right|^{2}=\frac{4 K_{\mathrm{sw}}}{\left|s_{1}\left(1+\sigma_{ \pm} Z_{0}\right)+s_{2}\right|^{2}} .
$$

Apart from a phenomenological factor $K_{\mathrm{sw}} \simeq 1$ addressed below, it describes the fraction of power transmitted through a dielectric slab containing an isotropic 2DES for a normally incident circularly polarized wave (plus and minus signs correspond to the right- and left-handed circular polarization, respectively). Two complex parameters $s_{1}=\cos (k d)-$ $i \epsilon^{-1 / 2} \sin (k d)$ and $s_{2}=\cos (k d)-i \sqrt{\epsilon} \sin (k d)$ describe the Fabry-Perót interference and are controlled by the product of the sample thickness $d=406 \mu \mathrm{m}$ and the wave number in the GaAs substrate, $k=\sqrt{\epsilon} \omega / c$. These parameters were accurately determined from the period of measured frequency dependence of transmittance at $B=0$, yielding the dielectric permittivity $\epsilon=12.06$ [60]. Apart from that, these $B=0$ measurements provided the value of the $\omega$-dependent factor $K_{\text {sw }}$ ( 0.913 for the fit in Fig. 1$)$ which is conventionally introduced to account for weak uncontrolled effects such as the remaining standing waves in the experimental cell $[60,62]$.
The magnetic field dependence of $\left|t_{ \pm}\right|^{2}$ in Eq. (1) is fully determined by the complex dynamic conductivity of the 2DES, $\sigma_{ \pm}$, which enters in combination with the impedance of free space $Z_{0}$ and is defined as $\sigma_{ \pm}=\sigma_{x x}(\omega, B) \pm i \sigma_{x y}(\omega, B)$ in terms of components $\sigma_{x x}=\sigma_{y y}$ and $\sigma_{x y}=-\sigma_{y x}$ of the conductivity tensor. We have checked that all features of the measured $\left|t_{ \pm}\right|^{2}$ remain independent of the microwave power in the whole available range, which demonstrates that our measurements reflect the linear-response transport properties of the 2DES [60]. The linear conductivity is modeled [29,60] as a sum $\sigma_{ \pm}=\sigma_{ \pm}^{\mathrm{D}}+\delta \sigma_{ \pm}$of classical Drude conductivity

$$
\sigma_{ \pm}^{\mathrm{D}}=\frac{e n}{\mu^{-1} \pm i B-i B_{\mathrm{CR}}},
$$

where $B_{\mathrm{CR}}=\omega m_{\mathrm{CR}} / e$ denotes the position of the $\mathrm{CR}$, and an oscillatory quantum correction

$$
\delta \sigma_{ \pm}=2 \delta^{2} \cos \frac{2 \pi \omega}{\omega_{\mathrm{c}}} \operatorname{Re} \sigma_{ \pm}^{\mathrm{D}} .
$$

Here $\delta=\exp \left(-\pi / \mu_{\mathrm{q}}|B|\right)$ is the Dingle factor, with $\mu_{\mathrm{q}}$ the quantum mobility which parameterizes the disorder broadening of LLs. For a reliable comparison of the theoretical model with experiment, in the transmittance fit according to Eqs. (1)-(3), presented in Fig. 1, we use the electron mobility $\mu=2.1 \times 10^{6} \mathrm{~cm}^{2} / \mathrm{V} \mathrm{s}$ and density $n=6.6 \times 10^{11} \mathrm{~cm}^{-2} \mathrm{de}-$ termined from the DC magnetotransport measurements [60].

The remaining three fitting parameters $B_{\mathrm{CR}}, m=e B / \omega_{\mathrm{c}}$, and $\mu_{\mathrm{q}}$ can be independently extracted from the transmittance data. Namely, the $\mathrm{CR}$ position $B_{\mathrm{CR}}$ can be accurately determined from the position of deep minimum in Fig. 1(b), where $\left|t_{+}\right|^{2}$ approaches zero in view of a large value of en $\mu Z_{0} \simeq 84$ representing $\sigma_{+} Z_{0}$ at $B=B_{\mathrm{CR}}$ [see Eqs. (1) and (2)]. The corresponding cyclotron mass $m_{\mathrm{CR}}=0.073 m_{0}$ represents the collective cyclotron motion of electrons, which by virtue of the Kohn theorem [63] is insensitive to electronelectron interactions. In contrast, the quasiparticle effective mass $m=0.068 m_{0}$, determined from the period of $\omega / \omega_{c}$ magneto-oscillations in both the microwave transmittance and DC resistance, represents the LL spacing in the vicinity of the Fermi level where the microwave-assisted scattering processes take place. This mass should therefore be sensitive to the Fermi-liquid renormalizations and can significantly differ from $m_{\mathrm{CR}}$ as confirmed by previous observations of MIRO in several materials [23,64-66]. Our data involve a large number of oscillation periods in both $\delta R$ and $\left|t_{+}\right|^{2}$ and thus enable a precise determination of $m$. The results presented in Fig. 1 demonstrate that the periods of MIRO and of quantum oscillations [Eq. (3)] in the dynamic conductivity coincide. Taking into account that, unlike MIRO, quantum oscillations in transmittance become more pronounced for lower transport mobility, this opens up an opportunity to explore the renormalization effects $[23,64-66]$ in a broader class of 2DESs.

The form of Eq. (3) suggests that knowledge of a single parameter $\mu_{\mathrm{q}}$ should be sufficient to reproduce not only the low- $B$ decay, but also the shape and magnitude of magnetooscillations in the relative quantum correction $\delta \sigma_{ \pm} / \operatorname{Re} \sigma_{ \pm}^{\mathrm{D}}$ to the dissipative part of dynamic Drude conductivity. Taking into account that the values of all other relevant parameters were fixed using the results of independent measurements [60], this makes the accuracy of the fit in Fig. 1(c) quite 
remarkable. The obtained value of $\mu_{\mathrm{q}}=0.23 \times 10^{6} \mathrm{~cm}^{2} / \mathrm{V} \mathrm{s}$ is approximately 10 times smaller than the transport mobility, which implies the dominance of small-angle impurity scattering typical for modern high-mobility 2DESs. The validity of the above description is additionally supported by fits of the transmittance data obtained for higher frequencies that yield similar values of $\mu_{\mathrm{q}}$ [60].

The same value of $\mu_{\mathrm{q}}$ describes reasonably well the low$B$ decay of MIRO in $\delta R$, which are modeled using the theoretical expression [34]

$$
\frac{\delta R}{R}=-A_{\omega} \frac{\omega}{\omega_{\mathrm{c}}} \delta^{2} \sin \frac{2 \pi \omega}{\omega_{\mathrm{c}}}
$$

[see the red line in Fig. 1(a)]. The factor $A_{\omega}$ here depends on several parameters including the radiation characteristics [49] and properties of 2DESs [60]. The estimates show that MIRO at relevant temperatures should be dominated by the inelastic mechanism [29,32]. The fit provided the required value of the microwave power of $0.2 \mathrm{~mW}$, which is consistent with the characteristics of implemented radiation sources [60]. A closer look at high harmonics of MIRO shows, however, that the value of $\mu_{\mathrm{q}}$ found from the transmittance oscillations underestimates the actual decay of MIRO. We found that MIRO can be well fitted using a shorter value of $\mu_{\mathrm{q}}=$ $0.135 \times 10^{6} \mathrm{~cm}^{2} / \mathrm{V} \mathrm{s}$, which in turn does not fit the magnitude and number of oscillations in the transmittance [60]. This unexpected discrepancy requires understanding and deserves additional studies.

\section{DISCUSSION}

While the relative amplitude $\sim 1 \%$ of the observed oscillations in $\left|t_{+}\right|^{2}$ is quite small, they represent a significant effect $\left(2 \delta^{2} \sim 0.5\right.$ at $\left.|B|=0.2 \mathrm{~T}\right)$ in the dissipative part of the dynamic conductivity $\operatorname{Re} \sigma_{+}$, which defines the fraction of the microwave power absorbed by the 2 DES. The reason is that outside an extremely narrow range $\left|B-B_{\mathrm{CR}}\right| \lesssim$ $\mu^{-1} \sim 0.05 \mathrm{~T}$ near the CR, the shape of the magnetotransmittance is controlled by the imaginary part of conductivity $\operatorname{Im} \sigma_{+} \simeq e n /\left(B_{\mathrm{CR}}-B\right)$, which describes classical forced oscillations of electrons in the microwave field in the absence of scattering and thus is insensitive to Landau quantization [60]. The real part, proportional to the rate of photon-assisted scattering off impurities, is modified as the thermally averaged product $\langle v(\varepsilon) v(\varepsilon+\hbar \omega)\rangle$ of initial and final densities of states for transitions between the disorder-broadened LLs. For the relevant high $T$, corresponding to suppressed Shubnikov-de Haas oscillations, and in the limit of strongly overlapping LLs $v(\varepsilon) / \nu_{0} \simeq 1-2 \delta \cos 2 \pi \varepsilon / \hbar \omega_{\mathrm{c}}$, where $\nu_{0}$ denotes the constant density of states at $B=0$, this average reduces to $v_{0}^{2}\left(1+2 \delta^{2} \cos 2 \pi \omega / \omega_{\mathrm{c}}\right)$, reproducing Eq. (3) $[4,29,60]$.

The approximation of overlapping LLs works well for $\delta \ll 1$, but underestimates the amplitude of magnetooscillations in Fig. 1 for $|B| \gtrsim 0.2 \mathrm{~T}$, corresponding to the transition to the regime of separated LLs. More importantly, the shape of magneto-transmittance at $|B| \gtrsim 0.2 \mathrm{~T}$ is also influenced by remaining uncontrolled effects such as the standing waves in the experimental cell [60]. These detri- mental effects are apparently the main cause of deviations of the fitting curve from measured $\left|t_{+}\right|^{2}$ both at $B \sim B_{\mathrm{CR}}$ and at $B \sim-B_{\mathrm{CR}}$, which makes difficult a reliable comparison between the obtained data and theory in these interesting ranges of $B$. Importantly, in the well-controlled range of $|B|<0.2 \mathrm{~T}$ illustrated in Fig. 1(c), we do not observe any deviations between the theoretical fit and data that are asymmetric with respect to the sign of $B$. This suggests that the polarization dependence of the measured transmittance $\left|t_{+}\right|^{2}$ and therefore of the dynamic conductivity is well captured by theory [29], as opposed to MIRO, where strong deviations were reported for GaAs-based 2DESs [10,49], while a recent experiment with electrons on the surface of liquid He demonstrated a polarization dependence consistent with the theory predictions [20].

Similar to the Shubnikov-de Haas oscillations in the DC transport response, quantum oscillations in transmittance discussed above are a direct manifestation of the Landau quantization and thus should emerge in any 2DES in the appropriate range of radiation frequencies and magnetic fields. Classical memory effects can be another potential source of strong $\omega / \omega_{\mathrm{c}}$ oscillations in high-frequency dissipative transport $[40,43,51,52]$ that can be studied using high-precision measurements of transmittance. Unlike universal quantum oscillations, the shape, phase, and damping of these classical oscillations are strongly sensitive to the type of random potential of impurities realized in a particular 2DES. One further interesting potential application concerns hydrodynamic and magnetoplasmon effects in high-frequency transport, which in particular are proposed $[53,54]$ to be responsible for a huge photoresistance peak observed at $\omega \simeq 2 \omega_{\mathrm{c}}[67,68]$.

\section{CONCLUSION}

We have observed resonant transmittance features at multiple integer harmonics of the cyclotron resonance which follow the microwave-induced resistance oscillations concurrently measured in DC magnetotransport. The detected transmittance oscillations, including their polarization dependence, accurately follow the theoretical predictions relating them to impurity- and photon-assisted transitions between distant Landau levels, thus supporting the quantum mechanisms proposed for the explanation of MIRO. Our work demonstrates that high-precision measurements of transmittance can be a versatile tool for studies of high-frequency dissipative transport of systems with moderately high mobility, in particular providing an opportunity to explore the renormalization effects $[23,64-66]$ in a broader class of two-dimensional materials.

\section{ACKNOWLEDGMENTS}

We acknowledge discussions with D. A. Kozlov and support from Austrian Science Funds (Grant No. I3456-N27), from the Russian Foundation for Basic Research (Grant No. 17-52-14007), from the German Research Foundation (DFG Project No. GA501/14-1), and from the Elite Network of Bavaria (Grant No. K-NW-2013-247). 
[1] M. A. Zudov, R. R. Du, J. A. Simmons, and J. L. Reno, Shubnikov-de Haas-like oscillations in millimeterwave photoconductivity in a high-mobility two-dimensional electron gas, Phys. Rev. B 64, 201311(R) (2001).

[2] R. G. Mani, J. H. Smet, K. von Klitzing, V. Narayanamurti, W. B. Johnson, and V. Umansky, Zero-resistance states induced by electromagnetic-wave excitation in GaAs/AlGaAs heterostructures, Nature (London) 420, 646 (2002).

[3] M. A. Zudov, R. R. Du, L. N. Pfeiffer, and K. W. West, Evidence for a New Dissipationless Effect in 2D Electronic Transport, Phys. Rev. Lett. 90, 046807 (2003).

[4] I. A. Dmitriev, A. D. Mirlin, D. G. Polyakov, and M. A. Zudov, Nonequilibrium phenomena in high Landau levels, Rev. Mod. Phys. 84, 1709 (2012).

[5] M. A. Zudov, I. V. Ponomarev, A. L. Efros, R. R. Du, J. A. Simmons, and J. L. Reno, New Class of Magnetoresistance Oscillations: Interaction of a Two-Dimensional Electron Gas with Leaky Interface Phonons, Phys. Rev. Lett. 86, 3614 (2001).

[6] C. L. Yang, J. Zhang, R. R. Du, J. A. Simmons, and J. L. Reno, Zener Tunneling between Landau Orbits in a High-Mobility Two-Dimensional Electron Gas, Phys. Rev. Lett. 89, 076801 (2002).

[7] W. Zhang, M. A. Zudov, L. N. Pfeiffer, and K. W. West, Resonant Phonon Scattering in Quantum Hall Systems Driven by dc Electric Fields, Phys. Rev. Lett. 100, 036805 (2008).

[8] W. Zhang, M. A. Zudov, L. N. Pfeiffer, and K. W. West, Resistance Oscillations in Two-Dimensional Electron Systems Induced by Both ac and dc Fields, Phys. Rev. Lett. 98, 106804 (2007).

[9] S. Wiedmann, G. M. Gusev, O. E. Raichev, A. K. Bakarov, and J. C. Portal, Microwave Zero-Resistance States in a Bilayer Electron System, Phys. Rev. Lett. 105, 026804 (2010).

[10] J. H. Smet, B. Gorshunov, C. Jiang, L. Pfeiffer, K. West, V. Umansky, M. Dressel, R. Meisels, F. Kuchar, and K. von Klitzing, Circular-Polarization-Dependent Study of the Microwave Photoconductivity in a Two-Dimensional Electron System, Phys. Rev. Lett. 95, 116804 (2005).

[11] S. I. Dorozhkin, L. Pfeiffer, K. West, K. von Klitzing, and J. H. Smet, Random telegraph photosignals in a microwave-exposed two-dimensional electron system, Nat. Phys. 7, 336 (2011).

[12] A. A. Bykov, I. V. Marchishin, A. V. Goran, and D. V. Dmitriev, Microwave induced zero-conductance state in a Corbino geometry two-dimensional electron gas with capacitive contacts, Appl. Phys. Lett. 97, 082107 (2010).

[13] D. Konstantinov, Y. Monarkha, and K. Kono, Effect of Coulomb Interaction on Microwave-Induced Magnetoconductivity Oscillations of Surface Electrons on Liquid Helium, Phys. Rev. Lett. 111, 266802 (2013).

[14] A. D. Levin, Z. S. Momtaz, G. M. Gusev, O. E. Raichev, and A. K. Bakarov, Microwave-Induced Magneto-Oscillations and Signatures of Zero-Resistance States in Phonon-Drag Voltage in Two-Dimensional Electron Systems, Phys. Rev. Lett. 115, 206801 (2015).

[15] S. I. Dorozhkin, A. A. Kapustin, V. Umansky, K. von Klitzing, and J. H. Smet, Microwave-Induced Oscillations in Magnetocapacitance: Direct Evidence for Nonequilibrium Occupation of Electronic States, Phys. Rev. Lett. 117, 176801 (2016).

[16] Q. Shi, M. A. Zudov, I. A. Dmitriev, K. W. Baldwin, L. N. Pfeiffer, and K. W. West, Fine structure of high-power microwave-induced resistance oscillations, Phys. Rev. B 95, 041403(R) (2017).

[17] M. A. Zudov, O. A. Mironov, Q. A. Ebner, P. D. Martin, Q. Shi, and D. R. Leadley, Observation of microwave-induced resistance oscillations in a high-mobility two-dimensional hole gas in a strained quantum well, Phys. Rev. B 89, 125401 (2014).

[18] D. F. Kärcher, A. V. Shchepetilnikov, Y. A. Nefyodov, J. Falson, I. A. Dmitriev, Y. Kozuka, D. Maryenko, A. Tsukazaki, S. I. Dorozhkin, I. V. Kukushkin, M. Kawasaki, and J. H. Smet, Observation of microwave induced resistance and photovoltage oscillations in $\mathrm{MgZnO} / \mathrm{ZnO}$ heterostructures, Phys. Rev. B 93, 041410(R) (2016).

[19] R. Yamashiro, L. V. Abdurakhimov, A. O. Badrutdinov, Y. P. Monarkha, and D. Konstantinov, Photoconductivity Response at Cyclotron-Resonance Harmonics in a Nondegenerate TwoDimensional Electron Gas on Liquid Helium, Phys. Rev. Lett. 115, 256802 (2015).

[20] A. A. Zadorozhko, Y. P. Monarkha, and D. Konstantinov, Circular-Polarization-Dependent Study of Microwave-Induced Conductivity Oscillations in a Two-Dimensional Electron Gas on Liquid Helium, Phys. Rev. Lett. 120, 046802 (2018).

[21] M. Otteneder, I. A. Dmitriev, S. Candussio, M. L. Savchenko, D. A. Kozlov, V. V. Bel'kov, Z. D. Kvon, N. N. Mikhailov, S. A. Dvoretsky, and S. D. Ganichev, Sign-alternating photoconductivity and magnetoresistance oscillations induced by terahertz radiation in HgTe quantum wells, Phys. Rev. B 98, 245304 (2018).

[22] B. Friess, I. A. Dmitriev, V. Umansky, L. Pfeiffer, K. West, K. von Klitzing, and J. H. Smet, Acoustoelectric Study of Microwave-Induced Current Domains, Phys. Rev. Lett. 124, 117601 (2020).

[23] D. Tabrea, I. A. Dmitriev, S. I. Dorozhkin, B. P. Gorshunov, A. V. Boris, Y. Kozuka, A. Tsukazaki, M. Kawasaki, K. von Klitzing, and J. Falson, Microwave response of interacting oxide two-dimensional electron systems, Phys. Rev. B 102, 115432 (2020).

[24] E. Mönch, D. A. Bandurin, I. A. Dmitriev, I. Y. Phinney, I. Yahniuk, T. Taniguchi, K. Watanabe, P. Jarillo-Herrero, and S. D. Ganichev, Observation of terahertz-induced magnetooscillations in graphene, Nano Lett. 20, 5943 (2020).

[25] P. Kumaravadivel, M. T. Greenaway, D. Perello, A. Berdyugin, J. Birkbeck, J. Wengraf, S. Liu, J. H. Edgar, A. K. Geim, L. Eaves, and R. K. Kumar, Strong magnetophonon oscillations in extra-large graphene, Nat. Commun. 10, 3334 (2019).

[26] M. L. Savchenko, M. Otteneder, I. A. Dmitriev, N. N. Mikhailov, Z. D. Kvon, and S. D. Ganichev, Terahertz photoresistivity of a high-mobility 3D topological insulator based on a strained HgTe film, Appl. Phys. Lett. 117, 201103 (2020).

[27] V. I. Ryzhii, Photoconductivity characteristics in thin films subjected to crossed electric and magnetic fields, Sov. Phys. Solid State 11, 2078 (1970).

[28] A. C. Durst, S. Sachdev, N. Read, and S. M. Girvin, RadiationInduced Magnetoresistance Oscillations in a 2D Electron Gas, Phys. Rev. Lett. 91, 086803 (2003).

[29] I. A. Dmitriev, A. D. Mirlin, and D. G. Polyakov, CyclotronResonance Harmonics in the ac Response of a 2D Electron Gas with Smooth Disorder, Phys. Rev. Lett. 91, 226802 (2003).

[30] A. V. Andreev, I. L. Aleiner, and A. J. Millis, Dynamical Symmetry Breaking as the Origin of the Zero-dc-Resistance State in an ac-Driven System, Phys. Rev. Lett. 91, 056803 (2003). 
[31] M. G. Vavilov and I. L. Aleiner, Magnetotransport in a twodimensional electron gas at large filling factors, Phys. Rev. B 69, 035303 (2004).

[32] I. A. Dmitriev, M. G. Vavilov, I. L. Aleiner, A. D. Mirlin, and D. G. Polyakov, Theory of microwave-induced oscillations in the magnetoconductivity of a two-dimensional electron gas, Phys. Rev. B 71, 115316 (2005).

[33] M. G. Vavilov, I. L. Aleiner, and L. I. Glazman, Nonlinear resistivity of a two-dimensional electron gas in a magnetic field, Phys. Rev. B 76, 115331 (2007).

[34] I. A. Dmitriev, M. Khodas, A. D. Mirlin, D. G. Polyakov, and M. G. Vavilov, Mechanisms of the microwave photoconductivity in two-dimensional electron systems with mixed disorder, Phys. Rev. B 80, 165327 (2009).

[35] O. E. Raichev, Magnetic oscillations of resistivity and absorption of radiation in quantum wells with two populated subbands, Phys. Rev. B 78, 125304 (2008).

[36] Y. Monarkha and D. Konstantinov, Magneto-oscillations and anomalous current states in a photoexcited electron gas on liquid helium, J. Low Temp. Phys. 197, 208 (2019).

[37] I. A. Dmitriev, Self-oscillations and noise-induced flips of spontaneous electric field in microwave-induced zero resistance state, Europhys. Lett. 126, 57004 (2019).

[38] M. T. Greenaway, R. Krishna Kumar, P. Kumaravadivel, A. K. Geim, and L. Eaves, Magnetophonon spectroscopy of Dirac fermion scattering by transverse and longitudinal acoustic phonons in graphene, Phys. Rev. B 100, 155120 (2019).

[39] O. E. Raichev and M. A. Zudov, Effect of Berry phase on nonlinear response of two-dimensional fermions, Phys. Rev. Research 2, 022011(R) (2020).

[40] I. A. Dmitriev, A. D. Mirlin, and D. G. Polyakov, Oscillatory ac conductivity and photoconductivity of a two-dimensional electron gas: Quasiclassical transport beyond the Boltzmann equation, Phys. Rev. B 70, 165305 (2004).

[41] A. D. Chepelianskii and D. L. Shepelyansky, Microwave stabilization of edge transport and zero-resistance states, Phys. Rev. B 80, 241308(R) (2009).

[42] S. A. Mikhailov, Theory of microwave-induced zero-resistance states in two-dimensional electron systems, Phys. Rev. B 83, 155303 (2011).

[43] Y. M. Beltukov and M. I. Dyakonov, Microwave-Induced Resistance Oscillations as a Classical Memory Effect, Phys. Rev. Lett. 116, 176801 (2016).

[44] G. Abstreiter, J. P. Kotthaus, J. F. Koch, and G. Dorda, Cyclotron resonance of electrons in surface space-charge layers on silicon, Phys. Rev. B 14, 2480 (1976).

[45] T. Ando, Theory of cyclotron resonance lineshape in a twodimensional electron system, J. Phys. Soc. Jpn. 38, 989 (1975).

[46] S. A. Studenikin, M. Potemski, A. Sachrajda, M. Hilke, L. N. Pfeiffer, and K. W. West, Microwave-induced resistance oscillations on a high-mobility two-dimensional electron gas: Exact waveform, absorption/reflection and temperature damping, Phys. Rev. B 71, 245313 (2005).

[47] A. Wirthmann, B. D. McCombe, D. Heitmann, S. Holland, K.-J. Friedland, and C.-M. Hu, Far-infrared-induced magnetoresistance oscillations in $\mathrm{GaAs} / \mathrm{Al}_{x} \mathrm{Ga}_{1-x}$ As-based two-dimensional electron systems, Phys. Rev. B 76, 195315 (2007).

[48] L.-C. Tung, C. L. Yang, D. Smirnov, L. N. Pfeiffer, K. W. West, R. R. Du, and Y.-J. Wang, Submillimeter wave induced resistance oscillations in ultra-high mobility twodimensional electron systems, Solid State Commun. 149, 1531 (2009).

[49] T. Herrmann, I. A. Dmitriev, D. A. Kozlov, M. Schneider, B. Jentzsch, Z. D. Kvon, P. Olbrich, V. V. Bel'kov, A. Bayer, D. Schuh, D. Bougeard, T. Kuczmik, M. Oltscher, D. Weiss, and S. D. Ganichev, Analog of microwave-induced resistance oscillations induced in $\mathrm{GaAs}$ heterostructures by terahertz radiation, Phys. Rev. B 94, 081301(R) (2016).

[50] O. M. Fedorych, M. Potemski, S. A. Studenikin, J. A. Gupta, Z. R. Wasilewski, and I. A. Dmitriev, Quantum oscillations in the microwave magnetoabsorption of a two-dimensional electron gas, Phys. Rev. B 81, 201302(R) (2010).

[51] D. G. Polyakov, F. Evers, and I. V. Gornyi, Cyclotron resonance in antidot arrays, Phys. Rev. B 65, 125326 (2002).

[52] S. I. Dorozhkin, A. A. Kapustin, I. A. Dmitriev, V. Umansky, K. von Klitzing, and J. H. Smet, Evidence for non-Markovian electron dynamics in the microwave absorption of a twodimensional electron system, Phys. Rev. B 96, 155306 (2017).

[53] P. S. Alekseev and A. P. Alekseeva, Transverse Magnetosonic Waves and Viscoelastic Resonance in a Two-Dimensional Highly Viscous Electron Fluid, Phys. Rev. Lett. 123, 236801 (2019).

[54] V. A. Volkov and A. A. Zabolotnykh, Bernstein modes and giant microwave response of a two-dimensional electron system, Phys. Rev. B 89, 121410(R) (2014).

[55] A. D. Chepelianskii and D. L. Shepelyansky, Floquet theory of microwave absorption by an impurity in the two-dimensional electron gas, Phys. Rev. B 97, 125415 (2018).

[56] T. Baba, T. Mizutani, and M. Ogawa, Elimination of persistent photoconductivity and improvement in $\mathrm{Si}$ activation coefficient by Al spatial separation from Ga and $\mathrm{Si}$ in Al-Ga-As:Si solid system-A novel short period AlAs/n-GaAs superlattice, Jpn. J. Appl. Phys. 22, L627 (1983).

[57] K.-J. Friedland, R. Hey, H. Kostial, R. Klann, and K. Ploog, New Concept for the Reduction of Impurity Scattering in Remotely Doped GaAs Quantum Wells, Phys. Rev. Lett. 77, 4616 (1996).

[58] V. Umansky, M. Heiblum, Y. Levinson, J. Smet, J. Nübler, and M. Dolev, MBE growth of ultra-low disorder 2DEG with mobility exceeding $35 \times 10^{6} \mathrm{~cm}^{2} / \mathrm{V} \mathrm{s}$, J. Cryst. Growth $\mathbf{3 1 1}$, 1658 (2009).

[59] M. J. Manfra, Molecular beam epitaxy of ultra-high-quality AlGaAs/GaAs heterostructures: Enabling physics in lowdimensional electronic systems, Annu. Rev. Condens. Matter Phys. 5, 347 (2014).

[60] See Supplemental Material at http://link.aps.org/supplemental/ 10.1103/PhysRevResearch.3.L012013 for details of transmittance measurements, additional transmission data for different radiation frequencies, dependence of MIRO and transmittance on the radiation power and DC, magnetotransport in the absence and presence of radiation, measurements of MIRO using double-modulation technique, full expression for the amplitude of MIRO, summary of the fitting procedure including a table of obtained sample parameters, discussion of the low- $B$ decay of oscillations in transmission and MIRO, and conditions for observation of transmittance oscillations in high-mobility 2DESs.

[61] A. M. Shuvaev, G. V. Astakhov, C. Brüne, H. Buhmann, L. W. Molenkamp, and A. Pimenov, Terahertz magneto-optical 
spectroscopy in HgTe thin films, Semicond. Sci. Technol. 27, 124004 (2012).

[62] U. Dziom, THz spectroscopy of novel spin and quantum Hall systems, Ph.D. thesis, Vienna University of Technology, 2018.

[63] W. Kohn, Cyclotron resonance and de Haas-van Alphen oscillations of an interacting electron gas, Phys. Rev. 123, 1242 (1961).

[64] A. T. Hatke, M. A. Zudov, J. D. Watson, M. J. Manfra, L. N. Pfeiffer, and K. W. West, Evidence for effective mass reduction in GaAs/AlGaAs quantum wells, Phys. Rev. B 87, 161307(R) (2013).

[65] A. V. Shchepetilnikov, D. D. Frolov, Y. A. Nefyodov, I. V. Kukushkin, and S. Schmult, Renormalization of the effective mass deduced from the period of microwave-induced resistance oscillations in GaAs/AlGaAs heterostructures, Phys. Rev. B 95, 161305(R) (2017).

[66] X. Fu, Q. A. Ebner, Q. Shi, M. A. Zudov, Q. Qian, J. D. Watson, and M. J. Manfra, Microwave-induced resistance oscillations in a back-gated GaAs quantum well, Phys. Rev. B 95, 235415 (2017).

[67] Y. Dai, R. R. Du, L. N. Pfeiffer, and K. W. West, Observation of a Cyclotron Harmonic Spike in Microwave-Induced Resistances in Ultraclean GaAs/AlGaAs Quantum Wells, Phys. Rev. Lett. 105, 246802 (2010).

[68] A. T. Hatke, M. A. Zudov, L. N. Pfeiffer, and K. W. West, Giant microwave photoresistivity in high-mobility quantum Hall systems, Phys. Rev. B 83, 121301(R) (2011). 